\title{
Can Surgeons Identify ACL Femoral Ridges Landmark and Optimal Tunnel Position? A 3D Model Study
}

\author{
Carl Laverdiere, B.Eng., Drew Schupbach, M.D., Justin Schupbach, M.D., \\ Eric Harvey, B.Sc., Mathieu Boily, M.D., Mark Burman, M.D., and Paul A. Martineau, M.D.
}

\begin{abstract}
Purpose: To examine the ability of surgeons to identify the osseous landmarks associated with the femoral anterior cruciate ligament (ACL) footprint and locate optimal tunnel placement on 3-dimensional (3D) printed models compared with intraoperative placement. Methods: Twelve sports fellowship-trained orthopaedic surgeons were asked to identify a femoral landmark and an ACL footprint on 10 different 3D printed knees. The 3D models were made based on 20 real patients with different anatomical morphology who later received ACL reconstructive surgery using independent drilling. ImageJ software was used to quantify the measurements, which were then analyzed using descriptive statistics. Results: Overall, none of the surgeons were able to consistently identify the junction of the bony ridges. The mean error per participant ranged from 2.81 to $7.34 \mathrm{~mm}$ in the proximal direction $(P=3.30 \mathrm{e}-05)$ and from 2.42 to $8.05 \mathrm{~mm}$ in the posterior direction $(P=4.88 \mathrm{e}-12)$. None of the surgeons were able to appropriately identify the center of the femoral footprint on the anatomic 3D models. The difference between the center of the footprint surgeons identified on the 3D model and the tunnel graft location in surgery was significantly different $(P=.0046)$. On average, the magnitude of the error when the surgeons performed the actual surgery was $3.72 \pm 2.43 \mathrm{~mm}$, whereas on the $3 \mathrm{D}$ models it was $5.82 \pm 1.97$ mm. Conclusions: Experienced sports fellowship-trained orthopaedic surgeons were unable to correctly identify the junction of the intercondylar and bifurcate ridges and the native ACL footprint on 3D models. Operatively placed tunnels were more accurate implying that looking either through a scope or soft-tissue landmarks play a significant role in surgeons ACL footprint localization. Clinical Relevance: The graft position for ACL reconstruction plays an important role on the kinematics of the knee. This paper shows that soft tissue landmarks are needed to provide reliable reference points for reconstruction.
\end{abstract}

$\mathbf{T}$ he success of anterior cruciate ligament (ACL) reconstruction is multifactorial, depending on the type of graft, fixation technique, sex, and degenerative changes. The graft position also plays an important role on the kinematics of the knee. ${ }^{1}$ Cadaveric studies have shown femoral tunnel placement in the anatomic

From the Department of Orthopedic Surgery, McGill University Health Centre, Montreal, Canada.

The authors report that they have no conflicts of interest in the authorship and publication of this article. Full ICMJE author disclosure forms are available for this article online, as supplementary material.

Received November 28, 2019; accepted May 13, 2020.

Address correspondence to Carl Laverdiere, B.Eng., Department of Orthopedic Surgery, McGill University Health Centre, Montreal General Hospital, 1650 Cedar Avenue, Room A5-175.1, Montréal, QC, H3G 1A4, Canada. E-mail: carl.laverdiere@mail.mcgill.ca

Crown Copyright $(2) 2020$ Published by Elsevier on behalf of the Arthroscopy Association of North America. This is an open access article under the CC BY-

NC-ND license (http://creativecommons.org/licenses/by-nc-nd/4.0/).

2666-061X/191397

https://doi.org/10.1016/j.asmr.2020.05.008 footprint of the ACL results in knee kinematics closest to the intact knee. ${ }^{2}$ As reported by the MARS study, femoral tunnel malposition was rated as the most common technical failure by $80 \%$ of the surgeons. ${ }^{3}$ Of the 2 tunnel sites, variations in the femoral tunnel have a much larger effect on stability than tibial tunnel variations. ${ }^{4}$ Hutchinson et al. ${ }^{5}$ defined the intercondylar ridge or "residents ridge" as the landmark defining the most anterior border of the ACL femoral insertion. This was supplemented by the description of an osseous ridge between the anteromedial and posterolateral bundle's femoral insertion termed the bifurcate ridge. ${ }^{6}$ The intersection of the bifurcate ridge and the intercondylar ridge define the osseous landmarks of the femoral insertion. However well-defined the osseous landmarks are in the literature, visibility during arthroscopic surgery and anatomical variability have necessitated refining of localization techniques and utilization of soft-tissue landmarks. ${ }^{7-9}$ No matter how well described a landmark or technique is in the 
Table 1. Technical Specification of 3D Printer

\begin{tabular}{lc}
\hline \multicolumn{1}{c}{ Filament type } & PLA \\
\hline Filament diameter & $1.75 \mathrm{~mm}$ \\
Extruder temperature & $200^{\circ} \mathrm{C}$ \\
Build plate temperature & $60^{\circ} \mathrm{C}$ \\
Nozzle diameter & $0.40 \mathrm{~mm}$ \\
Resolution (primary layer height) & $0.1 \mathrm{~mm}$ \\
Top solid layer & 8 \\
Outer perimeter shells & 3 \\
Infill & $15 \%$, rectilinear pattern \\
\hline
\end{tabular}

literature, the ability of the surgeon to identify the landmark intraoperatively will always be a variable. The purpose of this study is to examine the ability of surgeons to identify the osseous landmarks associated with the femoral ACL footprint and locate optimal tunnel placement on 3-dimensional (3D) printed models compared with intraoperative placement. We hypothesized that the points for graft placement the surgeons identify on 3D osseous model will be as accurate as those identified intraoperatively.

\section{Material and Methods}

\section{Study Protocol}

Institutional review board approval (MUHC reference \#2019-5322) was obtained before the onset of this study. Based on history and physical examination, patients that were suspected to have an acute ACL tear were recruited by our research coordinator. Patients with previous knee pathology including previous surgery, previous ligamentous injury, inflammatory arthropathy, or osteoarthritis and patients with a suspected multiligamentous knee injury were excluded from the study. Twenty patients $(6$ females, 14 males) were recruited with an average age of $33.7 \pm 11.33$ years. The median age was 32 years. As part of the normal preoperative work-up, a conventional 2-dimensional (2D) magnetic resonance imaging (MRI) was performed to confirm the diagnosis of an acute ACL tear. Afterwards, a 3D MRI scan was performed on the injured knee to acquire high-resolution images. Those patients then
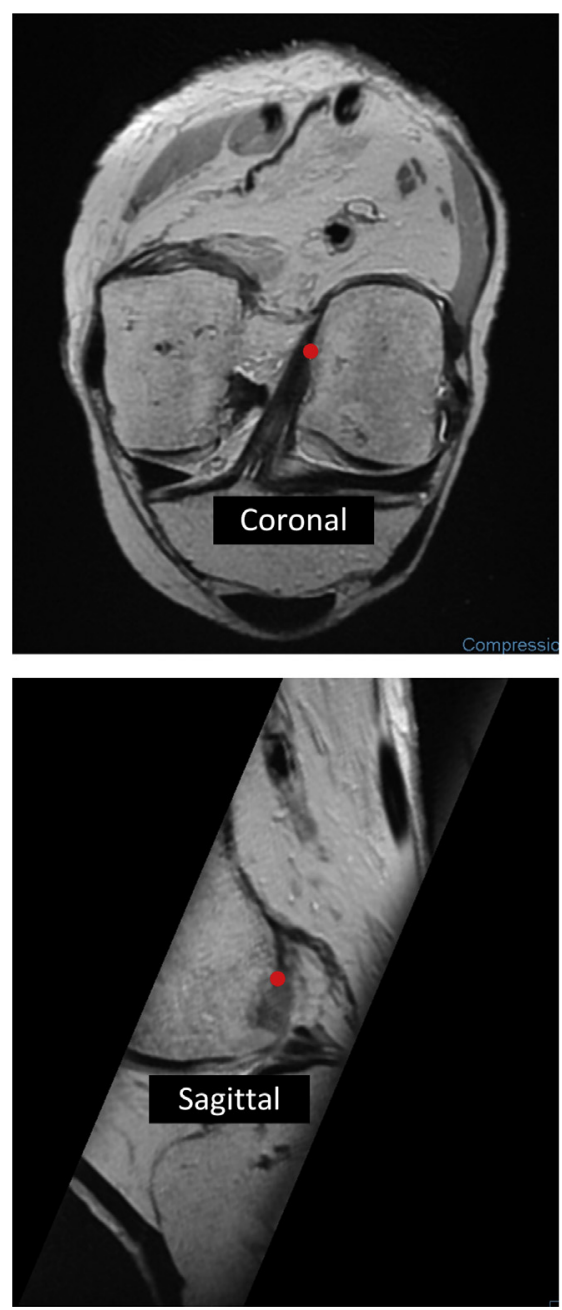
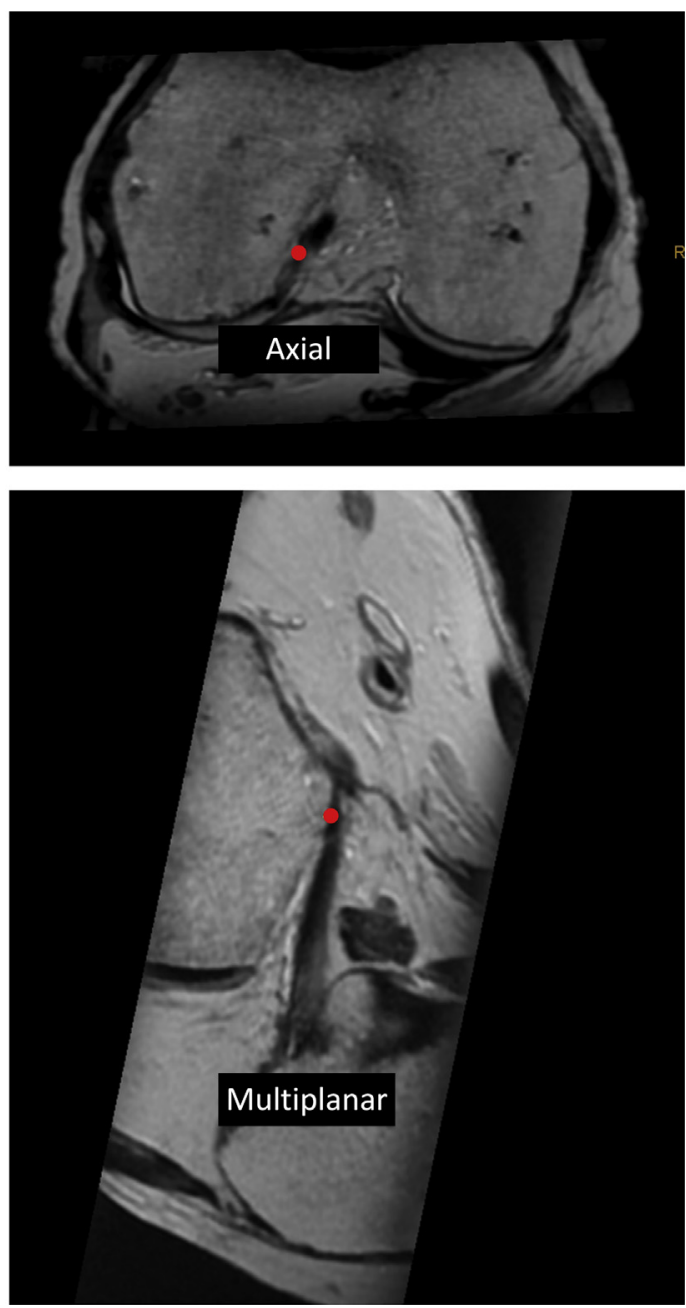

Fig 1. Visualization of the femoral footprint using 3dimensional magnetic resonance imaging on the lateral femoral condyle. 

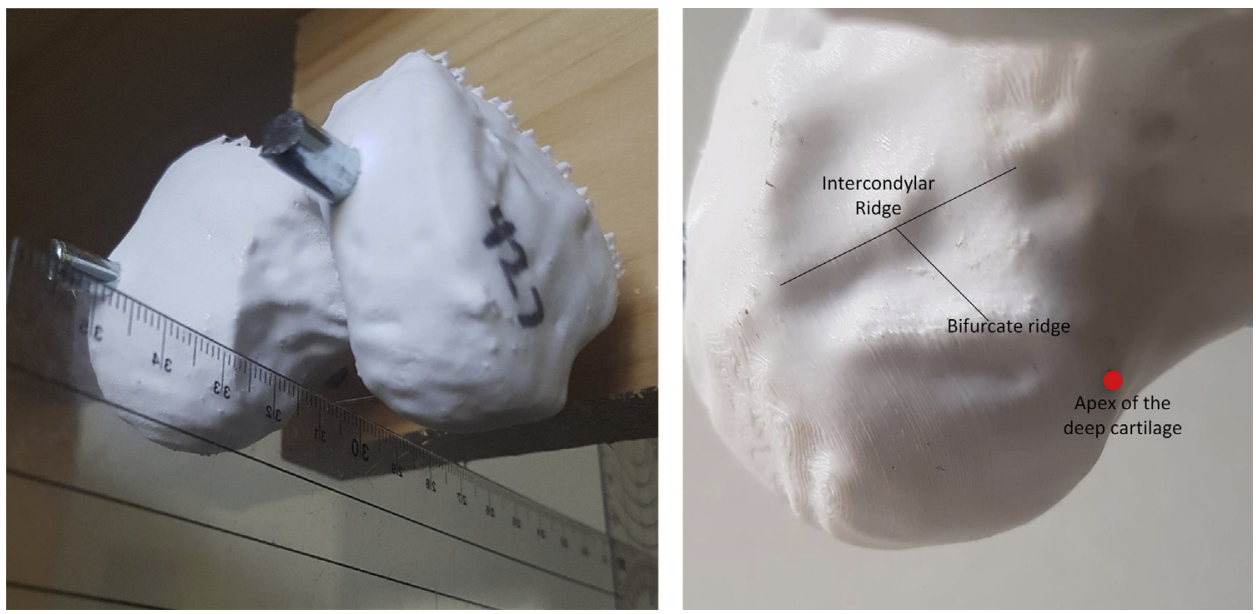

Fig 2. Identification of the intersection of the ridges and location of the center of the femoral footprint. Each model was positioned in the same orientation such that it represents a knee at $90^{\circ}$ in flexion. The ruler was used as a gauge for measurements, which were subsequently analyzed through the ImageJ software via a digital caliper.

underwent ACL reconstruction performed by 2 sports fellowship trained surgeon at our institution. Those 2 surgeons also participated in the model portion of the study but were blinded to the models. The surgical technique consisted of independent drilling with flexible or rigid reamers. A postoperative 3D MRI scan was acquired to evaluate the position of the graft. Distal femur models were then printed that duplicated the native anatomy to test other fellow surgeons.

The imaging protocol for this study was a previously validated isotropic 3D MRI protocol. ${ }^{10-12}$ Both 2D and 3D MRIs were performed using the same 1.5T TwinSpeed Excite high-definition MRI scanner (GE Medical Systems). In the scanner, the knees were positioned in near full extension, and an 8-channel high-definition surface coil was applied. Regarding the 3D MRI, an oblique-coronal proton density sequence along the plane of the ACL with slice gaps of $0.6 \mathrm{~mm} .{ }^{10,11}$ The mean scanning times for all 3 image acquisitions (2D MRI scan of injured knee and 3D MRI of bilateral knees) was 45 minutes.

\section{D Models Generation}

The 3D MRI Digital Imaging and Communications in Medicine scans were imported in 3D slicer software. ${ }^{13-17}$ This software enables segmentation of the Digital Imaging and Communications in Medicine scans to create a 3D model that mimics the native anatomy. Models were then printed using QidiTechl dual extruder 3D printer (QIDI TECH, Ruian, China). Technical information on the parameters use used can be found in Table 1. Using a previously validated reference point, ${ }^{18}$ the apex of the deep cartilage was identified on the 3D MRI for all subjects by a senior musculoskeletal radiologist. That same point was then marked on the 3D models to serve as a reference point for the measurements.
The use of 3D MRI allows for multiplanar reconstruction of the knee and does not limit to a single predefined viewing plane like standard 2D MRI. Thus, it is possible to observe the ACL along its course in the coronal-oblique plan. Multiplanar reconstructions and measurements were performed on a PACS workstation with embedded multiplanar software (InteleViewer; Intelerad Medical Systems). The boundaries of the native ACL of the uninjured knee and postoperative ACL graft were visualized with coronal-oblique and sagittal-oblique scans (Fig 1). Using the center of the footprints pre- and postoperatively, coordinates were defined in relation with the apex of the deep cartilage and recorded on all 3D MRI scans.

\section{Ridge and Femoral Footprint Analysis}

With a pin, 12 sports fellowship-trained orthopaedic surgeons were asked to identify the intersection between the intercondylar ridge and the bifurcate ridge on the 3D models (Fig 2). Afterwards, the surgeons were asked to identify the most anatomic femoral

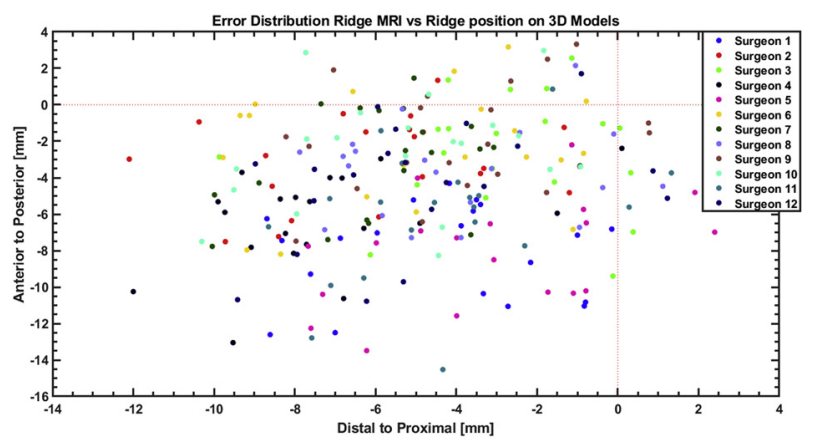

Fig 3. Error distribution of identified intersection of the intercondylar and bifurcate ridges by surgeons on 3D models. Each color represents a different person, with the $(0,0)$ point representing the intersection of the ridges on the MRI of the native knees. A negative value indicates a graft that is proximal and posterior. 
Table 2. Intersection of the Intercondylar Ridge and Bifurcate Identified by Surgeon and Radiologist on 3D Femoral Model vs Native

\begin{tabular}{|c|c|c|c|c|}
\hline & \multicolumn{2}{|c|}{ Mean Error of Ridge Position } & \multicolumn{2}{|c|}{$P$ Value } \\
\hline & Proximal-Distal Axis* (mm) & ${\text { Anteroposterior Axis }{ }^{\dagger}(\mathrm{mm})}$ & Proximal-Distal Axis* & Anteroposterior Axis \\
\hline Surgeon 1 & $-4.38 \pm 2.75$ & $-8.05 \pm 2.56$ & $2.19 \mathrm{e}-07$ & $1.47 \mathrm{e}-14$ \\
\hline Surgeon 2 & $-6.18 \pm 2.91$ & $-3.08 \pm 2.44$ & $2.38 \mathrm{e}-08$ & $5.20 \mathrm{e}-05$ \\
\hline Surgeon 4 & $-7.34 \pm 2.79$ & $-6.39 \pm 2.68$ & $1.62 \mathrm{e}-10$ & $1.44 \mathrm{e}-11$ \\
\hline Surgeon 5 & $-3.10 \pm 3.00$ & $-7.83 \pm 3.04$ & $1.35 \mathrm{e}-04$ & $1.49 \mathrm{e}-14$ \\
\hline Surgeon 6 & $-5.03 \pm 3.20$ & $-2.42 \pm 3.15$ & $2.71 \mathrm{e}-08$ & 0.0011 \\
\hline Surgeon 9 & $-3.99 \pm 2.66$ & $-1.88 \pm 2.94$ & $4.12 \mathrm{e}-05$ & 0.0101 \\
\hline Surgeon 10 & $-5.39 \pm 2.76$ & $-2.62 \pm 3.02$ & $3.44 \mathrm{e}-08$ & $3.01 \mathrm{e}-04$ \\
\hline Surgeon 11 & $-4.41 \pm 2.54$ & $-6.17 \pm 3.54$ & $6.39 e-07$ & $5.74 \mathrm{e}-11$ \\
\hline Surgeon 12 & $-4.95 \pm 2.94$ & $-4.37 \pm 3.36$ & $1.37 \mathrm{e}-07$ & $2.49 \mathrm{e}-07$ \\
\hline
\end{tabular}

*A positive value is defined as a graft that is purely distal relative to the native ACL.

${ }^{\dagger} \mathrm{A}$ positive value is defined as a graft that is purely anterior relative to the native ACL.

tunnel location on the same models (Fig 2). Measurements of those position were done with respect to the apex of the deep cartilage identified on the models. The measurements were performed by 2 separate observers to corroborate the measurements. Both observers were blinded to the measurements of the other observer. Because the 3D models were generated from MRI, reference measurements were performed on the MRI itself by a senior musculoskeletal radiologist. The center of the native femoral footprint was identified following a previously validated methodology. ${ }^{11}$ The intersection of the intercondylar and bifurcate ridges was also identified on MRI. The measurements performed on the MRI were used as the reference points.

\section{Statistical Analysis}

The number of patients required was predetermined using a power analysis to detect an error distance of 1.0 $\mathrm{mm}$, considering the average graft width is $8.0 \mathrm{~mm}$. The root-mean-square difference of ACL between contralateral knees were found to be $1.2 \mathrm{~mm}$ by Scanlan et al. ${ }^{19}$ Moreover, the mean error reported by Han et al. ${ }^{10}$ comparing measurements from 3D MRI to dissected specimens was $1.5 \mathrm{~mm}$. Thus, to obtain a standard error of less than $1.0 \mathrm{mmm}$, a minimum of 18 patients were needed $((3 \mathrm{~mm}+1.2 \mathrm{~mm}) / 1.0 \mathrm{~mm})^{2}$.

Descriptive statistics and the Student $t$ test were used to describe and compare the position identified by the surgeons on the 3D model relative the native structures. A Kruskal-Wallis l-way analysis of variance were then used to compare the error distances between the 12 surgeons based on the results of a Shapiro-Wilk test. All statistical analysis was done using Matlab software suite (MATLAB R2018a, The Mathworks, Natick, MA). A $P$ value of less than .05 was deemed statistically significant. The Intraclass correlation coefficient was used

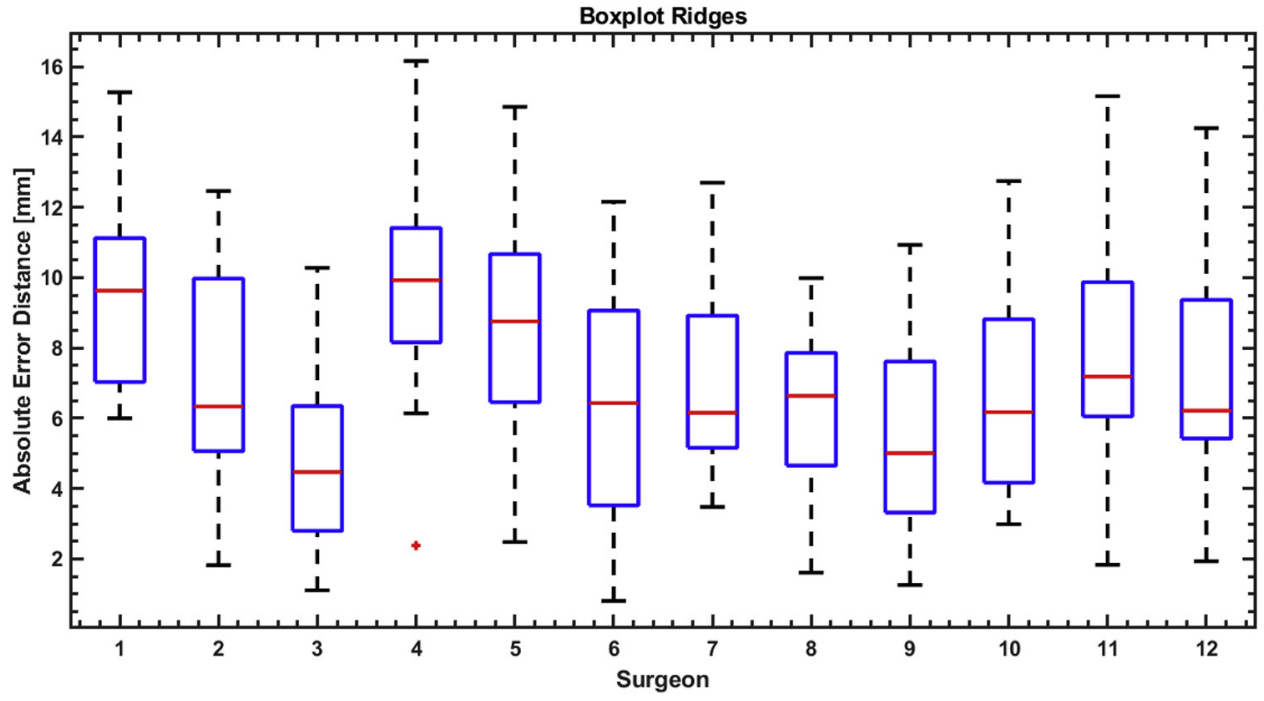

Fig 4. Box plot of error distance (3D model vs ridges) by each surgeon for the ridges. The central mark is the median, while the edges of the box are the 25th and 75 th percentiles. Error distance between surgeons is significantly different. 


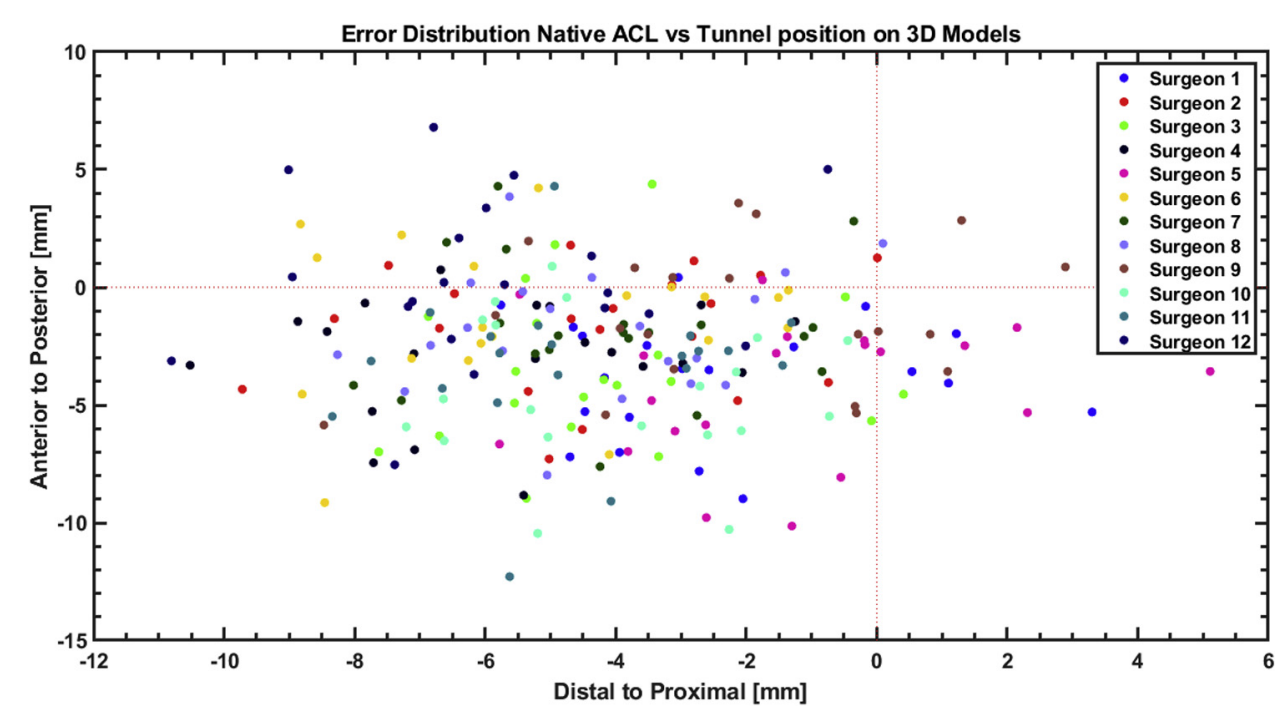

Fig 5. Error distribution of identified tunnel position by surgeons on 3D models. Each color represents a different surgeon, with the $(0,0)$ point representing the center of the femoral ACL footprint of the native knees. A negative value indicates a graft that is proximal and posterior.

to assess the agreement between both observers as defined by McGraw et al. ${ }^{20}$ Two independent observers performed the measurement. An intraclass coefficient of 0.955 in the distal-proximal axis and 0.961 in the anteroposterior axis shows excellent inter-rater agreement. The post hoc analysis yielded a power of 0.987 .

\section{Results}

A total of 12 sports fellowship-trained orthopaedic surgeons were recruited in this study. The surgeons were asked to identify the intersection of the intercondylar and bifurcate ridges on the anatomical 3D model. The error distribution of the points is shown in Fig 3. Overall, none of the surgeons were able to consistently identify the junction of the ridges $(P<.05$; Table 2), as the mean error per participant ranged from 2.81 to $7.34 \mathrm{~mm}$ in the proximal direction $(P=3.30 \mathrm{e}$ $05)$ and from 2.42 to $8.05 \mathrm{~mm}$ in the posterior direction $(P=4.88 \mathrm{e}-12)$. Comparing error distances among the
12 surgeons demonstrated significant differences using the Kruskal-Willis l-way analysis of variance $(P=2.31$ e-07) with a box plot (Fig 4$)$.

Surgeons were then asked to identify the optimal location for an anatomic ACL femoral tunnel. The overall error distribution is shown in Fig 5. Again, none of the surgeons were able to appropriately identify the center of the femoral footprint on the anatomic 3D models $(P<.05$; Table 3$)$. Only surgeon 9 and surgeon 12 were able to replicate the anatomic location in the anteroposterior axis, with a $P$ value of .0985 and .6624 , respectively. The mean error ranged from 1.32 to 5.95 $\mathrm{mm}$ in the proximal direction $(P=6.17 \mathrm{e}-08)$ and from 4.33 to $-0.32 \mathrm{~mm}$ in the posterior direction $(P=6.43 \mathrm{e}$ 06). Comparing the error difference among all surgeons using an analysis of variance demonstrated a significant difference $(P=.019)$ with a box plot (Fig 6).

The variation of the error from where the surgeons thought the footprint was on the 3D model compared

Table 3. Surgeon ACL Tunnel Placement vs Native ACL Footprint on 3D Femoral Model

\begin{tabular}{|c|c|c|c|c|}
\hline & \multicolumn{2}{|c|}{ Mean Error of Reconstructed Graft } & \multicolumn{2}{|c|}{$P$ Value } \\
\hline & Proximal-Distal Axis* (mm) & Anteroposterior Axis $^{\dagger}$ (mm) & Proximal-Distal Axis* & Anteroposterior Axis ${ }^{\dagger}$ \\
\hline Surgeon 1 & $-2.41 \pm 2.44$ & $-3.87 \pm 2.54$ & 0.0036 & $1.21 \mathrm{e}-06$ \\
\hline Surgeon 2 & $-4.36 \pm 2.50$ & $-1.77 \pm 2.59$ & $1.20 \mathrm{e}-06$ & 0.0164 \\
\hline Surgeon 3 & $-4.20 \pm 2.16$ & $-3.52 \pm 3.27$ & $4.74 \mathrm{e}-07$ & $4.63 e-05$ \\
\hline Surgeon 4 & $-5.69 \pm 2.49$ & $-3.00 \pm 2.46$ & $1.22 \mathrm{e}-07$ & $5.03 e-05$ \\
\hline Surgeon 5 & $-1.32 \pm 2.70$ & $-4.33 \pm 2.95$ & 0.0909 & $1.16 \mathrm{e}-07$ \\
\hline Surgeon 6 & $-5.26 \pm 2.56$ & $-1.35 \pm 3.17$ & $4.91 \mathrm{e}-08$ & 0.0469 \\
\hline Surgeon 7 & $-4.11 \pm 2.18$ & $-1.85 \pm 2.82$ & $3.12 \mathrm{e}-07$ & 0.0144 \\
\hline Surgeon 8 & $-4.39 \pm 2.15$ & $-1.88 \pm 2.67$ & $6.24 \mathrm{e}-07$ & 0.0083 \\
\hline Surgeon 9 & $-2.11 \pm 2.80$ & $-1.28 \pm 2.96$ & 0.0243 & 0.0985 \\
\hline Surgeon 10 & $-4.09 \pm 2.09$ & $-4.43 \pm 3.07$ & $7.63 e-07$ & $6.06 \mathrm{e}-08$ \\
\hline Surgeon 11 & $-4.66 \pm 2.02$ & $-3.36 \pm 3.21$ & $3.53 e-07$ & $1.33 \mathrm{e}-05$ \\
\hline Surgeon 12 & $-5.95 \pm 2.37$ & $0.32 \pm 3.47$ & $5.92 \mathrm{e}-10$ & 0.6624 \\
\hline
\end{tabular}

*A positive value is defined as a graft that is purely distal relative to the native ACL.

${ }^{\dagger}$ A positive value is defined as a graft that is purely anterior relative to the native ACL. 


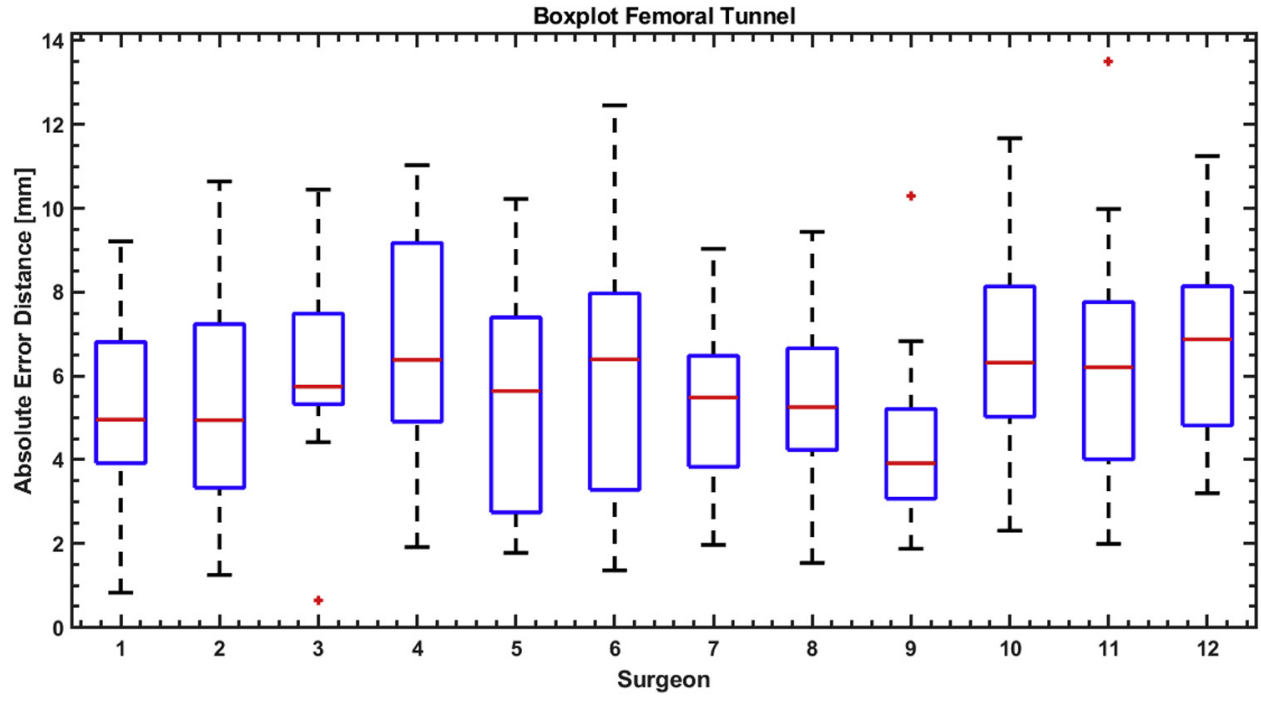

Fig 6. Box plot of error distance (3D model vs native footprint) by each surgeon for the femoral footprint. Error distance between surgeons is significantly different. with the surgically placed femoral tunnel is shown in Fig 7. Using an analysis of variance, from model to model, the error was significantly different for the femoral tunnel $(P=2.41 \mathrm{e}-19$; Fig 7$)$ and for the intersection of the ridges $(P=8.66 \mathrm{e}-17$; Fig 8$)$. This implies that some models yielded more accurate results than others. The overlay on Fig 7 represents the error performed by the surgeons performing the actual surgery. The difference between the center of the footprint surgeons identified on the 3D model and the tunnel graft location in surgery was significantly different $(P=.0046)$. On average, the magnitude of the error when the surgeons performed the actual surgery was $3.72 \pm 2.43 \mathrm{~mm}$, whereas on the 3D models, it was $5.82 \pm 1.97 \mathrm{~mm}$.

\section{Discussion}

The improved accuracy of intraoperative placement versus placement on the $3 \mathrm{D}$ model observed in this study supports that idea that soft tissues are used intraoperatively to aid surgeons in anatomic placement. Often, an ACL stump is present and may influence tunnel placement in anatomic reconstruction. Newer techniques using soft-tissue landmarks have been studied and validated such as measuring from the proximal articular cartilage. ${ }^{21}$ This method was further supported by recent reconstruction studies of the lateral femoral condyle, which showed it to be as reliable as the lateral intercondylar ridge. ${ }^{21}$ Much research has been done on osseous landmarks of the ACL footprint, but our study calls into question how accurately

Fig 7. Box plot of error distances by models for the femoral footprint by all participants. The orange dot represents the error performed by the surgeon during the real ACL surgery on the patient from which the models were made. Error distances between models is significantly different.

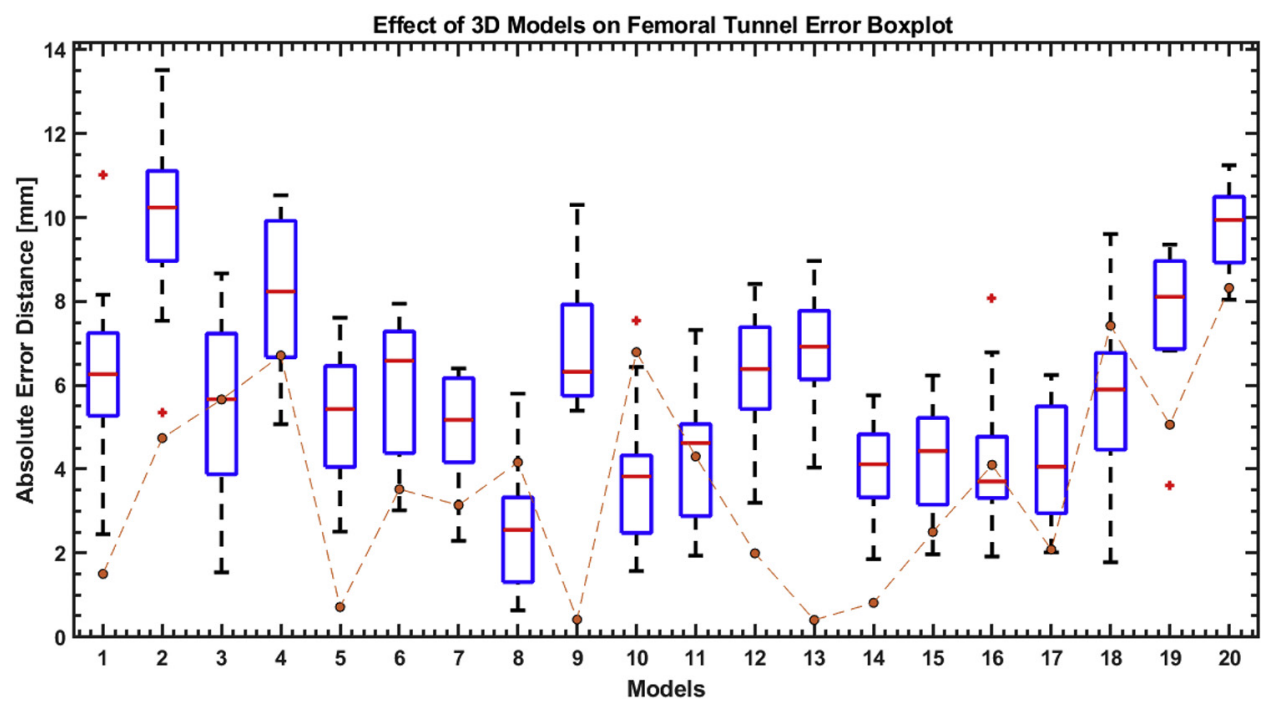


Fig 8. Box plot of error distances by models for the ridges by all participants. Error distances between models is significantly different.

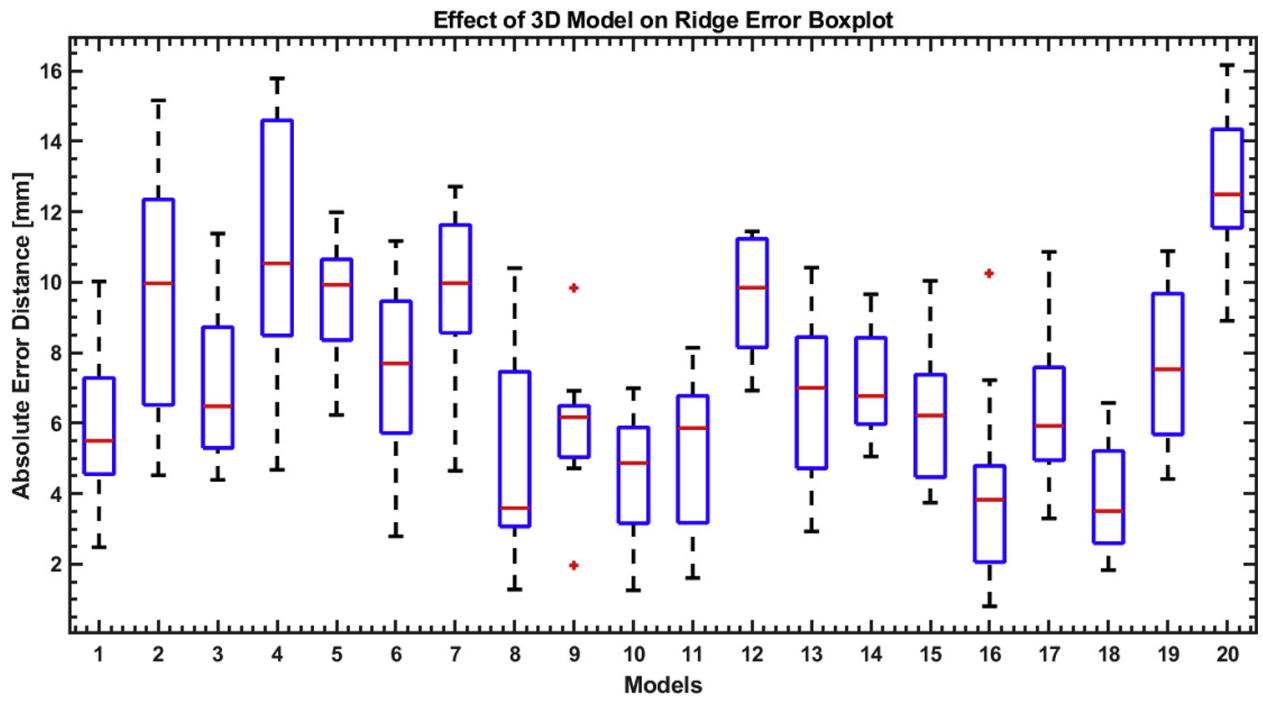

surgeons can identify the ridges. The surgeons consistently placed the femoral tunnels posterior and proximal to the femoral footprint on the 3D models. However, the actual tunnel placement measured on postoperative MRI was closer to the center of the footprint than the tunnel placement on the bony 3D models. This could be due to surgeons reverting to using posterior landmarks on the 3D models in the absence of soft-tissue landmarks. Therefore, the bony ridges may not be a consistent landmark in the absence of surrounding soft-tissue context.

A previous study performed using the same protocol was performed on 13 senior residents from 3 different Canadian orthopaedic residency programs. Interestingly, the error distribution was similar because most of the misses in both groups were in the proximal and posterior quadrant. All surgeons participating in the present study have done a sport fellowship training. This suggest that the soft tissues may have a greater importance than expected in locating the optimal position for femoral tunnel placement. Another piece of the answer may lie in the use of the scope and instruments in surgery may give a relative sense of the optimal position of the ACL graft.

\section{Limitations}

A few limitations exist that must be considered when interpreting the data of this study. The process for evaluating surgeons' ability to properly identify the intersection of femoral ridges and native ACL graft placement had possible drawbacks. The 3D printed models used only consisted of the bony landmarks with no connective tissue, cartilage, or ACL remnants to help with intraarticular orientation. It did not evaluate the ability of the surgeon to identify the ridges using a scope, which is the clinically relevant procedure.
Furthermore, the models were attached to an apparatus which did not allow knee movement to observe the joint from various aspects, which has been shown to impact graft positioning. ${ }^{22}$

\section{Conclusion}

Experienced sports fellowship-trained orthopaedic surgeons were unable to correctly identify the junction of the intercondylar and bifurcate ridges and the native ACL footprint on 3D models. Operatively placed tunnels were more accurate implying that looking either through a scope or soft-tissue landmarks play a significant role in surgeons ACL footprint localization.

\section{References}

1. Vignos MF, Kaiser JM, Baer GS, Kijowski R, Thelen DG. American Society of Biomechanics Clinical Biomechanics Award 2017: Non-anatomic graft geometry is linked with asymmetric tibiofemoral kinematics and cartilage contact following anterior cruciate ligament reconstruction. Clin Biomech 2018;56:75-83.

2. Musahl V, Plakseychuk A, VanScyoc A, et al. Varying femoral tunnels between the anatomical footprint and isometric positions - Effect on kinematics of the anterior cruciate ligament-reconstructed knee. Am J Sports Med 2005:33:712-718.

3. MARS Group, Wright RW, Huston LJ, Spindler KP, et al. Descriptive epidemiology of the Multicenter ACL Revision Study (MARS) cohort. Am J Sports Med 2010;38: 1979-1986.

4. Hefzy MS, Grood ES, Noyes FR. Factors affecting the region of most isometric femoral attachments: Part II: The anterior cruciate ligament. Am J Sports Med 1989;17: 208-216.

5. Hutchinson MR, Ash SA. Resident's ridge: Assessing the cortical thickness of the lateral wall and roof of the intercondylar notch. Arthroscopy 2003;19:931-935. 
6. Ferretti M, Ekdahl M, Shen W, Fu FH. Osseous landmarks of the femoral attachment of the anterior cruciate ligament: an anatomic study. Arthroscopy 2007;23:1218-1225.

7. Bhattacharyya R, Ker A, Fogg Q, Spencer SJ, Joseph J, Lateral Intercondylar ridge: Is it a reliable landmark for femoral ACL insertion? An anatomical study. Int J Surg 2018;50:55-59.

8. Steiner M. Anatomic single-bundle ACL reconstruction. Sports Med Arthrosc Rev 2009;17:247-251.

9. Van Eck CF, Martins CA, Vyas SM, Celentano U, van Dijk CN, Fu FH. Femoral intercondylar notch shape and dimensions in ACL-injured patients. Knee Surg Sports Traumatol Arthrosc 2010;18:1257-1262.

10. Han Y, Kurzencwyg D, Hart A, Powell T, Martineau PA. Measuring the anterior cruciate ligament's footprints by three-dimensional magnetic resonance imaging. Knee Surg Sports Traumatol Arthrosc 2012;20:986-995.

11. Hart A, Sivakumaran T, Burman M, Powell T, Martineau PA. A prospective evaluation of femoral tunnel placement for anatomic anterior cruciate ligament reconstruction using 3-dimensional magnetic resonance imaging. Am J Sports Med 2018;46:192-199.

12. Kijowski R, Davis KW, Woods MA, et al. Knee joint: Comprehensive assessment with 3D isotropic resolution fast spin-echo MR imaging-diagnostic performance compared with that of conventional MR imaging at 3.0 T. Radiology 2009;252:486-495.

13. Fedorov A, Beichel R, Kalpathy-Cramer J, et al. 3D Slicer as an image computing platform for the Quantitative Imaging Network. Magn Reson Imaging 2012;30:1323-1341.

14. Gering DT, Nabavi A, Kikinis R, et al. An integrated visualization system for surgical planning and guidance using image fusion and an open MR. J Magn Reson Imaging 2001;13:967-975.
15. Kikinis R, Pieper SD, Vosburgh KG. 3D Slicer: A platform for subject-specific image analysis, visualization, and clinical support. Intraoperative Imaging and Image-guided Therapy. Springer 2014:277-289.

16. Pieper S, Halle M, Kikinis R. 3D Slicer. Paper presented at: 2004 2nd IEEE International Symposium on Biomedical Imaging: Nano to Macro (IEEE Cat No. 04EX821), Arlington, VA; 2004, pp. 632-635 Vol. 1

17. Pieper S, Lorensen B, Schroeder W, Kikinis R. The NAMIC Kit: ITK, VTK, pipelines, grids and 3D slicer as an open platform for the medical image computing community. Paper presented at: 3rd IEEE International Symposium on Biomedical Imaging: Nano to Macro, 2006, Arlington, VA; 2006, pp. 698-701.

18. Hart A, Han Y, Martineau PA. The apex of the deep cartilage: A landmark and new technique to help identify femoral tunnel placement in anterior cruciate ligament reconstruction. Arthroscopy 2015;31:1777-1783.

19. Scanlan SF, Lai J, Donahue JP, Andriacchi TP. Variations in the three-dimensional location and orientation of the ACL in healthy subjects relative to patients after transtibial ACL reconstruction. J Orthop Res 2012;30:910-918.

20. McGraw KO, Wong SP. Forming inferences about some intraclass correlation coefficients. Psychol Methods 1996;1:30.

21. Bird JH, Carmont MR, Dhillon M, et al. Validation of a new technique to determine midbundle femoral tunnel position in anterior cruciate ligament reconstruction using 3-dimensional computed tomography analysis. Arthroscopy 2011;27:1259-1267.

22. Hoshino Y, Nagamune K, Yagi M, et al. The effect of intraoperative knee flexion angle on determination of graft location in the anatomic double-bundle anterior cruciate ligament reconstruction. Knee Surg Sports Traumatol Arthrosc 2009;17(9):1052-1060. 